\title{
Guiding Role of Industry Design Concept in Fitness Equipment Product Design
}

\author{
Zhao Yibo ${ }^{1, *}$ and Lu Zhengdao ${ }^{2, *}$ \\ ${ }^{1}$ Hebei Normal University, Shijiazhuang, China; ${ }^{2}$ Sports Institute, Lang Fang Teachers University, Langfang, China
}

\begin{abstract}
This study provides an understanding of the relationship between industrial design, biomechanics and mechanics. The main structure of this study is focused on: examining the outdoor fitness equipment's mechanism, analysing the mechanism's concurrency with the human movement, and based on this, designing an appropriate mechanism. Based on the basic theory, instrumental effect, main methods and knowledge database on industry design concept, this paper explores the application principles, effectiveness and innovative design along with the experimental methods of industry design concept in the field of family exercise equipment designing. And through the innovative design example of leg stretching equipment, the experimental methods have been verified for its effectiveness, which provides an important reference for the application of the industry design concept in the exercise equipment designing field.
\end{abstract}

Keywords: Fitness equipment product, guiding role, human movement, industry design concept.

\section{INTRODUCTION}

It is broadly accepted that regular physical activity can reduce the risk of chronic diseases such as obesity, heart disease, stroke, and high cholesterol [1]. Unfortunately, social trends indicate a gradual decrease in physical activity both at work and at home. Even leisure time activity, which is holding steady or just slightly increasing, can't overcome the overall decrease in the society's physical activity.

To compensate for the reduced physical activity in our daily lives, an increasing number of people are using exercise to offset this decline, which has created a growing demand for home gym and commercial gym exercise equipment [2]. Most of these machines require a user interface to make selections, such as stationary bikes, treadmills, elliptical cross trainers, and stair climbers. Traditionally, these machines have used membrane switch interfaces with simplified LED displays that provided time, distance, heart rate information, and an exercise profile. In commercial applications these machines have evolved to include LCD TV's for user entertainment, yet many have maintained their membrane switch technology as the user interface of choice. Typical interfaces that use membrane switches can include a user profile selection area, level and incline adjustments, a numerical pad for entering user information, an LED screen for displaying exercise session information, and a separate or integrated TV controller [3].

When the LCD TV and user interface modules are separate devices, mounting, cabling, and enclosures for up to three separate modules is required, which can increase manufacturing costs and affect equipment aesthetics and reliability.

*Address correspondence to these authors at the Åbo Akademi University, P.O. Box 311, FIN-65101 Vasa, Finland; Tel: +358-6-3247476; Fax: +3586-3247457; E-mails: jan.saarela@abo.fi, and fjalar.finnas@abo.fi
The concerns for product cost and improved industrial design have led many companies to explore touch enabling LCD screen using a software-driven graphical user interface (GUI) in order to combine the multiple modules into one, for an easy-to-use, cost-effective and aesthetically pleasing design [4]. This transition provides equipment manufacturers with increased design flexibility since buttons no longer have a single-purpose limitation and the usability of these machines can be greatly improved with an intuitive user interface that enhances the user experience.

Industrial design is a part of innovation. It makes its most significant contribution, as indicated above, in differentiation of similar products either by making them appear very different through colour and style, or by enhancing them with detail and minor additional features [5]. In more radical innovations where the new technology is involved, industrial design usually has a less central role because the novelty is provided by the new functionality. There are, of course, occasions where the new technology needs to be made more palatable to consumers and then the role of industrial design is more center stage. An example of this can be found in areas of medical technology, perhaps this is where patients need to be re-assured about the use of new machines and procedures.

If we return to the topic of the major contribution of industrial design, examples include the increasing variety of laptop computers, the increasing number of small consumer electronics for the home where colour and style are becoming the major differentiators, etc. [6]. In these ways, the use of industrial design does make a contribution to economic performance, and therefore, companies, investors and governments need to understand the importance of design expenditure for the innovation process. Design expenditure ranks with $R \& D$ expenditure as a strategic investment for companies. However, the lack of sophisticated techniques 
applied to design expenditure often holds back design investment. Project appraisal techniques and issues of finance and accounting methods need reconsideration [7]. For example, the finance techniques for valuing options used routinely in financial markets are rarely applied in the broader context. The benefits of design spending are systematically undervalued across companies and therefore the economy as a whole.

The rest of the paper is organized as follows. In the section 2, the critical application needs of exercise equipment are presented. Section 3 gives the theory of industry design concept. An application of industry design concept in exercise equipment design is given in Section 4. Some remarkable conclusions are listed in Section 5.

\section{CRITICAL APPLICATION NEEDS}

In the exercise equipment market, there are several highlevel needs that equipment manufacturers need to address when designing a new user interface for their exercise machines. They are: product robustness, ease of cleaning, optical clarity, interface accuracy and warranty. By addressing these end-customer needs for a robust system design, manufacturers can help ensure optimal system performance for the life of the exercise equipment.

\subsection{Product Robustness}

The most important application need is product robustness, which includes durability, reliability, and sealability. Being in a public environment, exercise machines are used repeatedly throughout the day, which results in numerous cleaning cycles, constant control interface use, contaminants from user perspiration, spilled water or sticky sports drinks, and constant mechanical vibrations. With most gyms operating 7 days a week and some gyms even operating 24/7, the ongoing use of the equipment can affect the reliability of the user interface. The most common user interface in this market relies on membrane switches for user input. The ASTM (American Society for Testing and Materials) defines membrane switches as momentary switch devices where at least one electrical contact is part of, or made of, a flexible substrate. A membrane switch consists of multiple layers of plastic films which are held together with adhesive layers. These films have conductive inks printed on opposing sides to perform the switching functions while the top surface is printed with a graphical design for displaying button functionality and improved aesthetics.

\subsection{Optical Clarity}

With high-definition LCD's now being embedded in exercise equipment control panels, the touch overlay should not degrade the LCD's visual quality and should provide maximum optical clarity, especially after numerous cleaning cycles. The polyester surface of a resistive touchscreen typically allows for $80 \%$ light transmission. However, due to on-going harsh cleaning habits of users the polyester surface can begin to show wear and reduced optical clarity due to micro-scratches collecting on the surface. This can result in a hazy appearance to the viewer due to refracting light when the light emitted by the underlying LCD display hits the transition points of each scratch. This refraction created by the surface scratches also reduces the light transmission exiting in this system. The result in that the actual light transmission can diminish over time with heavy use.

In addition to minimizing surface scratches, equipment manufacturers need control interfaces with anti-glare properties. Anti-glare surfaces are created with a surface treatment or chemical etching that is applied to a surface to reduce the glare from overhead lighting or sunlight from outside-facing windows typically found in commercial gyms. Without an anti-glare finish, the surface appears very shinny and reflective, increasing the glare and making the display difficult to read. Also, non anti-glare surfaces are more susceptible to finger prints. In this type of environment, an anti-glare finish is recommended to ensure optimal display viewing in indoor lighting conditions.

Industrial design studies function and form---and the connection between product, user, and environment. Generally, industrial design professionals work in small scale design, rather than overall design of complex systems such as buildings or ships. Industrial designers don't usually design motors, electrical circuits, or gearing that make machines move, but they may affect technical aspects through usability design and form relationships. Usually, they work with other professionals such as marketers to identify and fulfill customer needs and expectations.

Industrial design can overlap significantly with engineering design, and in different countries the boundaries of the two concepts can vary, but in general engineering focuses principally on functionality or 'utility of products' whereas industrial design focuses principally on 'aesthetic and user-interface aspects of products'. In many jurisdictions this distinction is effectively defined by credentials and/or licensure required to engage in the practice of engineering. "Industrial design" as such does not overlap much with the engineering sub-discipline of industrial engineering, except for the latter's sub-specialty of ergonomics.

Although the process of design may be considered "creative", many analytical processes also take place. In fact, many industrial designers often use various design methodologies in their creative process. Some of the processes that are commonly used are user research, sketching, comparative product research, model making, prototyping and testing. These processes are best defined by the industrial designers and/or other team members. Industrial designers often utilize 3D software, computeraided industrial design and CAD programs to move from concept to production. They may also build a prototype first and then use industrial $\mathrm{CT}$ scanning to test for interior defects and generate a CAD model. From this the manufacturing process may be modified to improve the product.

Industrial design may also focus on technical concepts, products, and processes. In addition to aesthetics, usability, and ergonomics, it can also encompass engineering, usefulness, market placement, and other concerns, such as psychology, desire, and the emotional attachment of the user. These values and accompanying aspects that form the basis of industrial design can vary between different schools of thought, and among practicing designers. 


\section{APPLICATION OF INDUSTRY DESIGN CONCEPT IN EXERCISE EQUIPMENT DESIGN}

\subsection{Application Analysis}

As one of important exercise equipments, leg stretching equipment belongs to a most basic device in learning dancing. The leg stretching equipment is a most basic and indispensable training device. When stretching leg muscles and ligaments, the training intensity for muscles is very hard. Every muscle is an organ consisting of muscle belly, tendon and the surrounding nerves, blood vessels and lymphatic vessels, etc., therefore, the muscles shall be stretched within scientific and safe range. And injuries resulted from over intensity, angle reversal and too hard stretching shall be avoided. At present, the ordinary leg stretching equipments are designed in single pole, see Fig. (1).

Although the existing equipments are simply made and widely used, accidents frequently occur because of over intensity, rotation angle reversal, too hard stretching and lack of installing controls, etc. and bring unpredictable harm to employed persons as well as fitness enthusiasts. Therefore, developing the equipment by adopting industry design concept not only has strong significance in reality, but also has very important significance in theory to develop and research products in relevant fields.

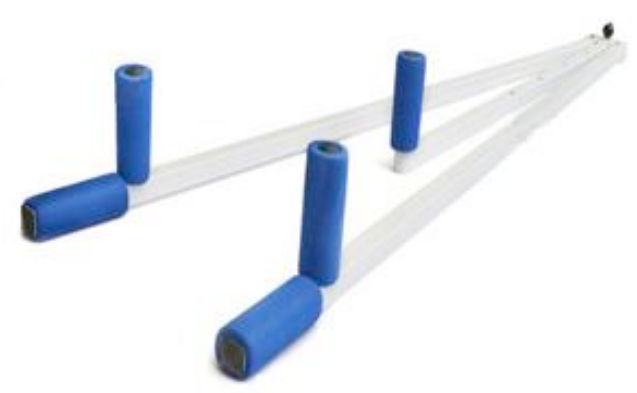

Fig. (1). The existing leg stretching equipment.

\subsection{Technical Contradiction Analysis of Engineering Parameters}

According to the analysis based on requirements of consumers, the consumer requirements of leg stretching equipment refer to users' objective needs on this product, including the needs of size, volume, appearance and operation, etc. Therefore, requirements of consumers (RC) facing product innovative design can be expressed as a function with all these elements as its variables:

$$
R C=f(S, V, A, O, \ldots)
$$

Based on the formulation, any change of any elements in RC may result in change of consumer requirements, thus bringing the corresponding technical contradictions. In accordance with the engineering parameters in $\mathrm{RC}$, problems of the existing equipment can be optimized and solved through project design, but at the same time it will result in technical contradictions under consumer requirements.

In the process of exercising muscles, relaxing legs and side opening ligaments, all the functions of the equipment need to be realized. Under the guarantee of safety (according to the general body data in Table 1), through designing a safety wheel axle to avoid reversal, adjust the scale of opening torque and separate exerciser's legs slowly in valid time to highly strengthen the flexibility of muscles and to avoid injuring muscles and deforming legs. "The length of stationary object" is described into No. 4 parameter in accordance with the general engineering parameters of RC.

\subsection{Construction of Technical Matrix of Contradictions}

After determining the description of engineering parameters, the appropriate principles of invention should be located through matrix of contradictions. As is shown in Table 2, the inventive principles in serial numbers have been achieved.

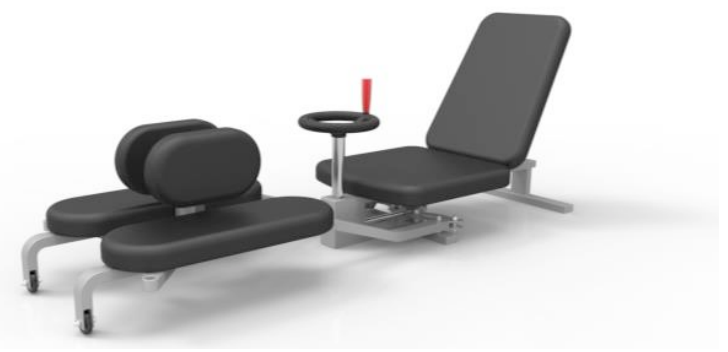

Fig. (2). Project design of leg stretching equipment for dancing.

\subsection{Improvement of Design Project in Accordance with Industry Design Principles}

The inventive principles achieved through matrix of contradictions have important implications for guidance and universality, but for the specific project and design. A practical analysis according to features of the product itself is still needed so as to achieve a specific solution for a specific problem. Through the author's careful analysis and filtering, the proper project for improving the product design is found from the above inventive principles, which can be described as follows:

(a) According to No.11 inventive principle "compensate object's relatively low reliability by adopting the preprepared emergency measures" and No. 8 inventive principle "weight compensation", the installation of bearing safety device can overcome the shortcomings that exist in the existing leg stretching equipments: in lacking of bearing safety device, strains often occur because of leg reversal when the range of motion is increased. What's more, this device can greatly decrease the viscosity of leg muscles and increase the speed of muscle contradiction and relaxation so as to avoid the strains of muscle.

(b) According to No.1 inventive principle "separation, namely separating an object into an independent group through collapsing and disassembling", design the main structure and devices of leg stretching equipment in modular manner. For example, designing the back cushion, side cushion for legs and separating device in detachable or assembled way can not only reduce the floor space in usage and bring the effect of convenience, but also can save space when putting it away or storing it and be operated handily.

By integrating the design elements that have been improved by industry design principles, the project of family 
Table 1. Value of 17-21 year-old girls control parts in national standard (Unit:cm).

\begin{tabular}{|c|c|c|c|}
\hline & Height & Cervical Height & Waist Height \\
\hline \hline Average & 162.2 & 37.8 & 100.0 \\
\hline Standard deviation & 5.80 & 5.58 & 4.48 \\
\hline Minimum & 150.0 & 123.1 & 89.0 \\
\hline Maximum & 173.2 & 150.2 & 109.8 \\
\hline Range & 23.2 & 27.1 & 20.8 \\
\hline
\end{tabular}

Table 2. Matrix of contradictions.

\begin{tabular}{|c|c|c|c|}
\hline \multirow{2}{*}{ Parameters Causing Deterioration } & \multicolumn{3}{|c|}{ Parameters for Improvement } \\
\cline { 2 - 4 } & 4 Length of Object & 6 Volume of Object & 12 form \\
\hline \hline 2 weight & $10,1,29,35$ & $35,30,13,2$ & $35,4,15$ \\
\hline 10 force & 28,1 & $1,8,36,37$ & $35,35,40$ \\
\hline 11 pressure & $1,14,35$ & $10,15,36,37$ & $22,1,18,4$ \\
\hline 13 stability & 37 & 39 & 35 \\
\hline
\end{tabular}

leg stretching equipments used for dancing is designed, as is shown in Fig. (2).

\section{CONCLUSION}

Through the testing and evaluation of CATIA and Rhino software, it shows that the equipment not only improves the shortcoming of the existing products, but also promotes the relevant needs of users. The project achieves such features of basic functions as safe usage, small floor space, convenient storage, good divisibility in appearance, jumpiness and vividness in feeling. The experimental results show that the improved design project of exercise equipment based on industry design principles is feasible. The specific process of the project is as follows: on the basis on functional analysis, the inventive methods of setting out contradiction matrix are searched to achieve the technical effects between the overall function and sub-functions and to filter the variable design elements; concerning the existing problems, make innovations, improvements and designs by applying various creative techniques; find variants to variable elements as many as possible by adopting principle of matrix and build a morphological matrix table; combine the variants and get a required innovative project of exercise equipment that adapts to the practical needs.

\section{CONFLICT OF INTEREST}

The authors confirm that this article content has no conflict of interest.

\section{ACKNOWLEDGEMENTS}

This work is supported by Hebei Province Sports Bureau 2014 annual sports scientific research project (20141019), the 2014 annual Hebei Provincial Association of Social Sciences Research (2014030803), the 13th batch of teaching reform project of Hebei Normal University.

\section{REFERENCES}

[1] P. Hekkert, D. Snelders, and P.C.W. van Wieringen, "Most advanced, yet acceptable': typicality and novelty as joint predictors of aesthetic preference in industrial design", British Journal of Psychology, vol. 94, pp. 111-124, 2003.

[2] R. Srinivasan, and A. Kraslawski, "Application of the TRIZ creativity enhancement approach to designinherently safer chemical processes", Chemical Engineering and Processing, vol. 4, pp. 507514, 2006

[3] E.R. Smith, and G.R. Semin, "Socially situated cognition in its social context", Advances in Experimental Social Psychology, vol. 36, pp. 53-117, 2004.

[4] J. Tooby, L. Cosmides, "Does beauty build adapted minds? Toward an evolutionary theory of aesthetics, fiction and the arts", Substance, vol. 94, pp. 6-27, 2011.

[5] X. Zhang, X. Mao, and S.M. Abourizk, "Developing a knowledge management system for improved value engineering practices in the construction industry", Automation in Construction, vol. 18, pp. 777-789, 2009.

[6] Y. Zhenhe and L. Xin, "Model evaluation based on emotional furniture industrial design elements", International Journal of Advancements in Computing Technology, vol. 22, pp. 73-78, 2012.

[7] Z. Zheng, and J. Zhang, "Model for evaluating the industrial design with interval grey linguistic variables", International Journal of Digital Content Technology and its Applications, vol. 6, pp. 136142,2012 